\title{
Recent Results and Status of TEXONON Experiments
}

\author{
Venktesh Singh* and Henry T. Wong \\ (on behalf of the TEXONO Collaboration) \\ Institute of Physics, Academia Sinica, Taipei 11529, Taiwan \\ E-mailivsingh@phys.sinica.edu.twintwong
}

\begin{abstract}
This article reviews the research program and efforts for the TEXONO Collaboration on neutrino and astro-particle physics. The "flagship" program is on reactorbased neutrino physics at the Kuo-Sheng (KS) Power Plant in Taiwan. A limit on the neutrino magnetic moment of $\mu_{\overline{\nu_{\mathrm{e}}}}<1.3 \times 10^{-10} \mu_{\mathrm{B}}$ at $90 \%$ confidence level was derived from measurements with a high purity germanium detector. Other physics topics at KS, as well as the various $R \& D$ program, are discussed.
\end{abstract}

\section{Introduction and History}

The TEXONO ${ }^{1}$ Collaboration [i] $\left[\begin{array}{l}1 \\ 1\end{array}\right]$ has been built up since 1997 to initiate and pursue an experimental program in Neutrino and Astroparticle Physics [2]. The Collaboration comprises more than 40 research scientists from major institutes/universities in Taiwan (Academia Sinica $^{\dagger}$, Chung-Kuo Institute of Technology, Institute of Nuclear Energy Research, National Taiwan University, National Tsing Hua University, and Kuo-Sheng Nuclear Power Station), China (Institute of High Energy Physics ${ }^{\dagger}$, Institute of Atomic Energy ${ }^{\dagger}$, Institute of Radiation Protection, Nanjing University, Tsing Hua University) and the United States (University of Maryland), with AS, IHEP and IAE (with ${ }^{\dagger}$ ) being the leading groups. It is the first research collaboration of this size and magnitude among scientists from Taiwan

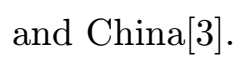

Results from recent neutrino experiments strongly favor neutrino oscillations which

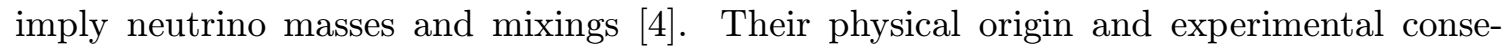
quences are not fully understood. There are strong motivations for further experimental efforts to shed light on these fundamental questions by probing standard and anomalous neutrino properties and interactions. The results can constrain theoretical models which will be necessary to interpret the future precision data. In addition, these studies would also explore new detection channels to open up new avenues of investigations.

\footnotetext{
${ }^{*}$ Speaker.

${ }^{1}$ Taiwan EXperiment On NeutrinO
} 
a)

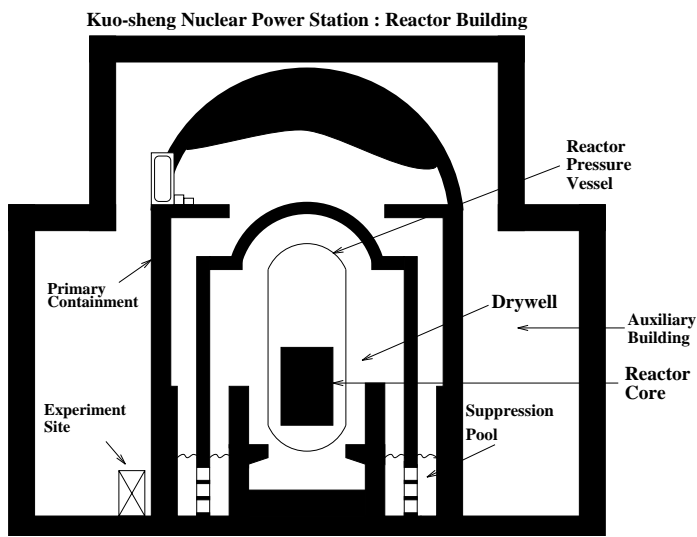

b)

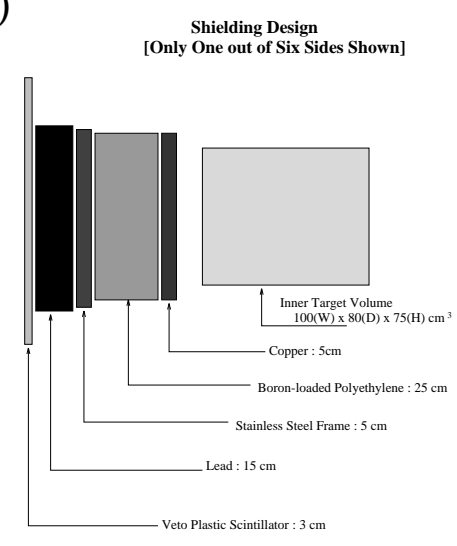

Figure 1: (a) Schematic side view, not drawn to scale, of the Kuo-Sheng Nuclear Power Station Reactor Building, indicating the experimental site. The reactor core-detector distance is about $28 \mathrm{~m}$. (b) Schematic layout of the inner target space, passive shieldings and cosmic-ray veto panels. The coverage is $4 \pi$ but only one face is shown.

The TEXONO research program is based on the the unexplored and unexploited theme of adopting detectors with high-Z nuclei, such as solid state device and scintillating crystals, for low-energy low-background experiments in Neutrino and Astroparticle Physics [5] main effort is a reactor neutrino experiment at the Kuo-Sheng (KS) Nuclear Power Station in Taiwan to study low energy neutrino properties and interactions [6i]. The Kuo-Sheng experiment is the first particle physics experiment in Taiwan. In parallel to the reactor experiment, various $\mathrm{R} \& \mathrm{D}$ efforts coherent with the theme are initiated and pursued.

Subsequent sections highlight the results and status of the program.

\section{Kuo-Sheng Neutrino Laboratory}

The "Kuo-Sheng Neutrino Laboratory" is located at a distance of $28 \mathrm{~m}$ from the core \#1 of the Kuo-Sheng Nuclear Power Station at the northern shore of Taiwan['히. A schematic view is depicted in Figure iina.

A multi-purpose "inner target" detector space of $100 \mathrm{~cm} \times 80 \mathrm{~cm} \times 75 \mathrm{~cm}$ is enclosed by $4 \pi$ passive shielding materials which have a total weight of 50 tons. The shielding provides attenuation to the ambient neutron and gamma background, and consists of, from inside out, $5 \mathrm{~cm}$ of OFHC copper, $25 \mathrm{~cm}$ of boron-loaded polyethylene, $5 \mathrm{~cm}$ of steel, $15 \mathrm{~cm}$ of lead, and cosmic-ray veto scintillator panels. The schematic layout of one side is shown in Figure

Different detectors can be placed in the inner space for the different scientific goals. The detectors are read out by a versatile electronics and data acquisition systems [i] based on a 16-channel, $20 \mathrm{MHz}, 8$-bit Flash Analog-to-Digital-Convertor (FADC) module. The readout allows full recording of all the relevant pulse shape and timing information for as long as several $\mathrm{ms}$ after the initial trigger. Software procedures have been devised to extend the effective dynamic range from the nominal 8-bit measurement range provided

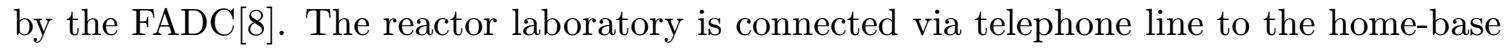




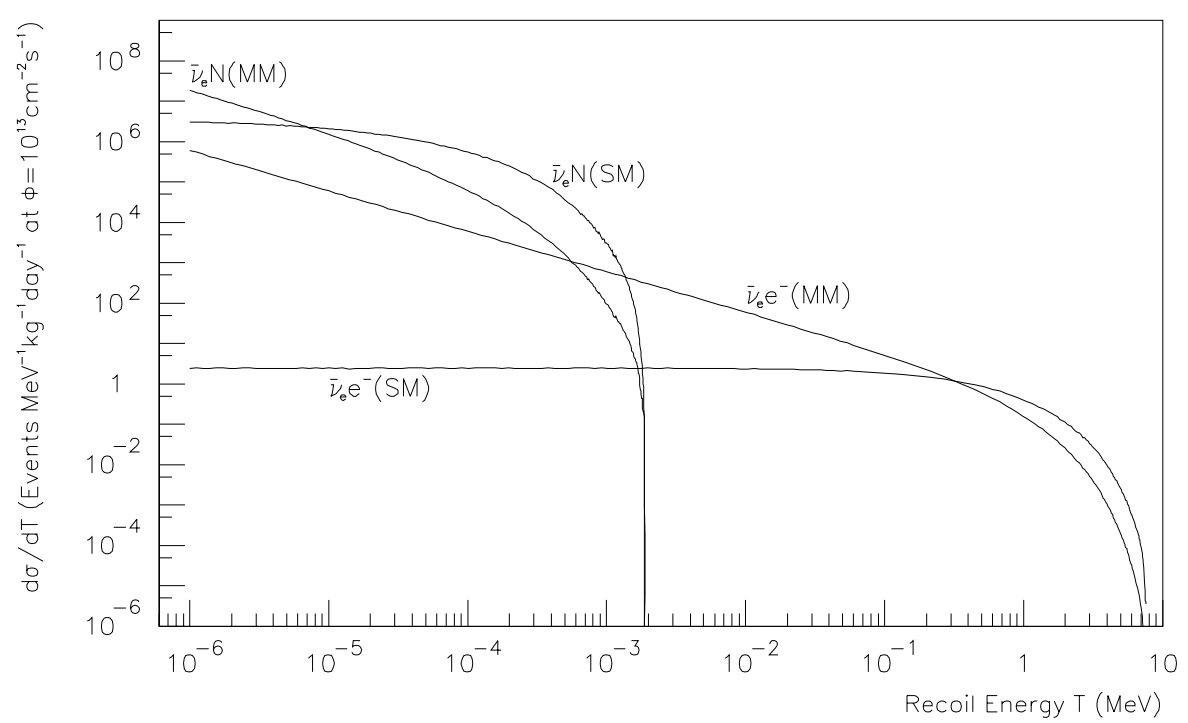

Figure 2: Differential cross section showing the recoil energy spectrum in $\overline{\nu_{e}}$-e and coherent $\overline{\nu_{e}}$-N scatterings, at a reactor neutrino flux of $10^{13} \mathrm{~cm}^{-2} \mathrm{~s}^{-1}$, for the Standard Model (SM) processes and due to a neutrino magnetic moment (MM) of $10^{-10} \mu_{\mathrm{B}}$.

laboratory at AS, where remote access and monitoring are performed regularly. Data are stored and accessed via the PC IDE-bus from a cluster of multi-disks arrays each with 800 Gbyte of memory.

The measure-able nuclear and electron recoil spectra due to reactor $\overline{\nu_{e}}$ are depicted in Figure $\overline{2}$, showing the effects due to Standard Model $[\sigma(\mathrm{SM})]$ and magnetic moment $\left[\sigma\left(\mu_{\nu}\right)\right]$ $\overline{\nu_{e}}$-electron scatterings $[\overline{9} \bar{j}]$, as well as the Standard Model neutrino coherent scatterings on the nuclei $[\sigma(\mathrm{coh})]$. It was recognized recently[i] $\left.{ }_{0}^{0}\right]$ that due to the uncertainties in the modeling of the low energy part of the reactor neutrino spectra, experiments to measure $\sigma(\mathrm{SM})$ with reactor neutrinos should focus on higher electron recoil energies $(\mathrm{T}>1.5 \mathrm{MeV})$, while $\mu_{\nu}$ searches should base on measurements with $\mathrm{T}<100 \mathrm{keV}$. Observation of $\sigma(\mathrm{coh})$ would require detectors with sub-keV sensitivities.

Accordingly, data taking were optimized for with these strategies. An ultra lowbackground high purity germanium (ULB-HPGe) detector was used for Period I (June 2001 till May 2002) data taking, while $186 \mathrm{~kg}$ of $\mathrm{CsI}(\mathrm{Tl})$ crystal scintillators were added in for Period II (starting January 2003). Both detector systems operate in parallel with the same data acquisition system but independent triggers. The target detectors are housed in a nitrogen environment to prevent background events due to the diffusion of the radioactive radon gas.

\subsection{Germanium Detector}

As depicted in Figure $13 \mathrm{i}$, the ULB-HPGe is surrounded by $\mathrm{NaI}(\mathrm{Tl})$ and $\mathrm{CsI}(\mathrm{Tl})$ crystal scintillators as anti-Compton detectors, and the whole set-up is further enclosed by another $3.5 \mathrm{~cm}$ of OFHC copper and lead blocks. 


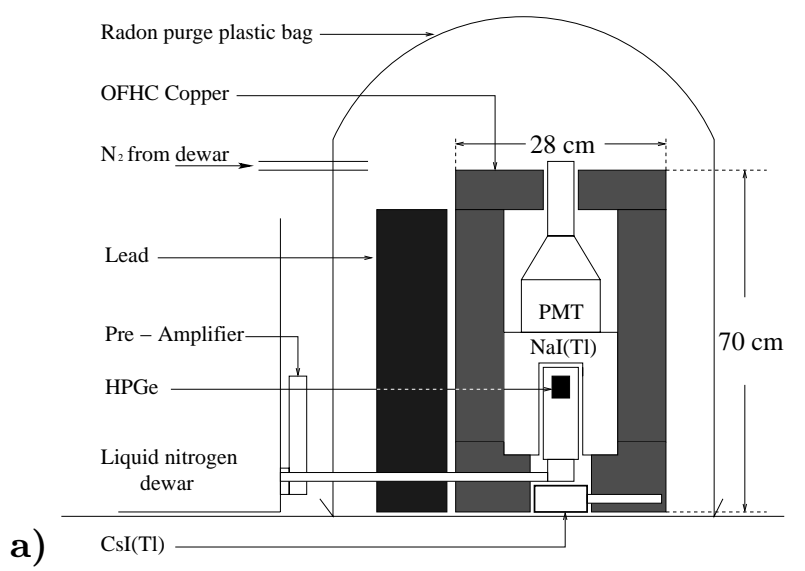

b)

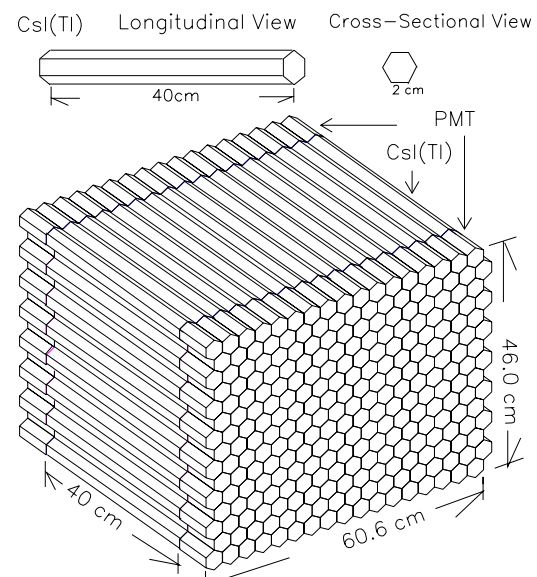

Figure 3: Schematic drawings of (a) the ULB-HPGe detector with its anti-Compton scintillators and passive shieldings; and (b) the $\mathrm{CsI}(\mathrm{Tl})$ target configuration where a total of 93 modules $(186 \mathrm{~kg})$ is installed for Period II.
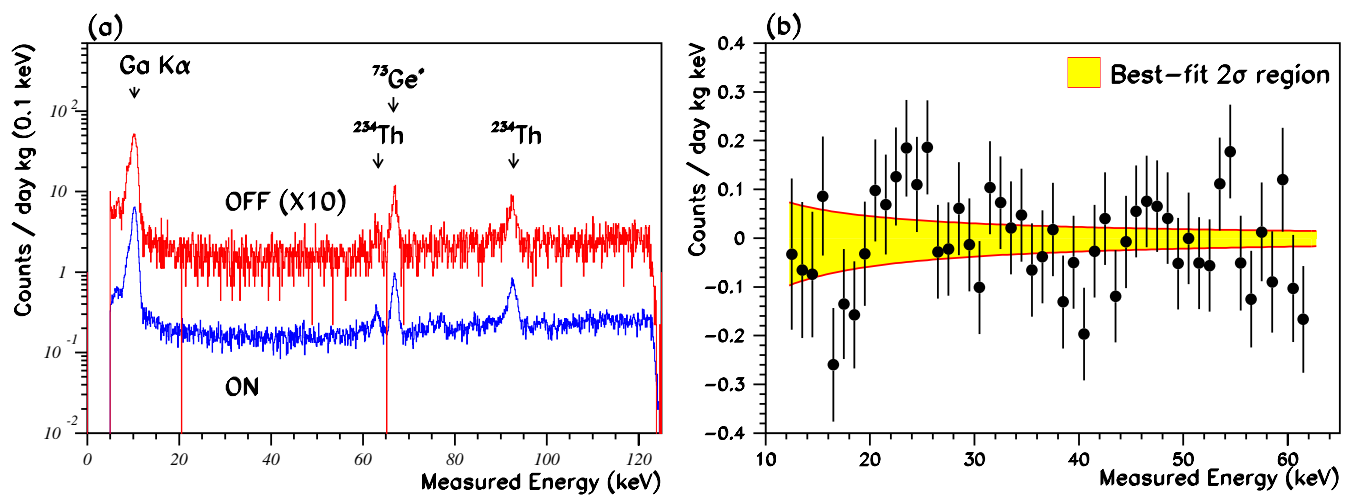

Figure 4: (a) The ON and OFF spectra after all identifiable background suppressed, for 4720 and 1250 hours of data, respectively. (b) The residual of the ON spectrum over the OFF background, with the $2-\sigma$ best-fit region overlaid.

After suppression of cosmic-induced background, anti-Compton vetos and convoluted events by pulse shape discrimination, the measured spectra for 4712/1250 hours of Reac-

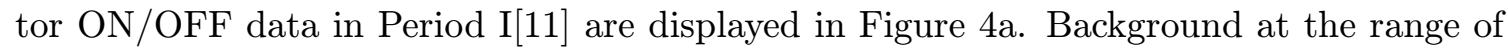
$1 \mathrm{keV}^{-1} \mathrm{~kg}^{-1}$ day $^{-1}$ and a detector threshold of $5 \mathrm{keV}$ are achieved. These are the levels comparable to underground Dark Matter experiment. Comparison of the ON and OFF spectra shows no excess and limits of the neutrino magnetic moment $\mu_{\overline{\nu_{\mathrm{e}}}}<1.3(1.0) \times 10^{-10} \mu_{\mathrm{B}}$ at 90(68)\% confidence level (CL) were set. The residual plot together with the best-fit regions are depicted in Figure i4⿴囗十

Depicted in Figure threshold in reactor experiments. The dotted lines denote the $\mathrm{R}=\sigma(\mu) / \sigma(\mathrm{SM})$ ratio at a particular $\left(\mathrm{T}, \mu_{\overline{\nu_{\mathrm{e}}}}\right)$. The $\mathrm{KS}(\mathrm{Ge})$ experiment has a much lower threshold of $12 \mathrm{keV}$ compared to the other measurements. The large R-values imply that the KS results are robust against the uncertainties in the SM cross-sections. The neutrino-photon couplings probed by $\mu_{\nu^{-}}$ searches in $\nu$-e scatterings are related to the neutrino radiative decays $\left(\Gamma_{\nu}\right)\left[\overline{1} \overline{2}_{2} \bar{i}\right]$. The indirect 

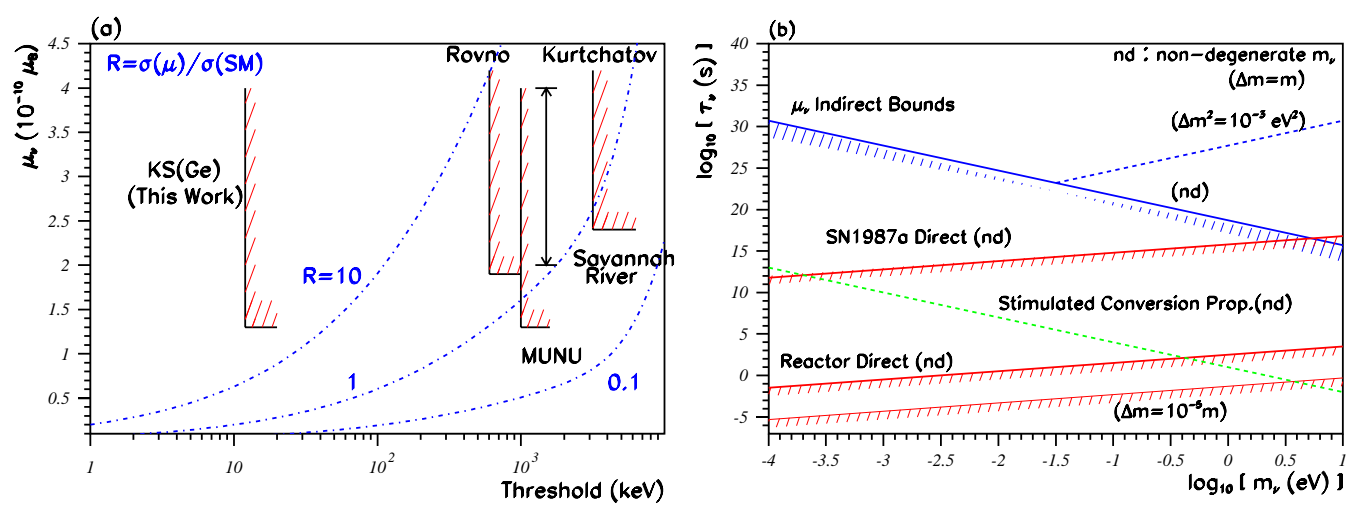

Figure 5: Summary of the results in (a) the searches of neutrino magnetic moments with reactor neutrinos, and (b) the bounds of neutrino radiative decay lifetime.

bounds on $\Gamma_{\nu}$ can be inferred and displayed in Figure give much more stringent bounds than the direct approaches.

The KS data with ULB-HPGe are the lowest threshold data so far for reactor neutrino experiments, and therefore allow the studies of several new and more speculative topics. Nuclear fission at reactor cores also produce electron neutrino $\left(\nu_{e}\right)$ through the production of unstable isotopes, such as ${ }^{51} \mathrm{Cr}$ and ${ }^{55} \mathrm{Fe}$, via neutron capture, The subsequent decays of these isotopes by electron capture would produce mono-energetic $\nu_{e}$. A realistic neutron transfer simulation has been carried out to estimate the flux. Physics analysis on the $\mu_{\nu}$ and $\Gamma_{\nu}$ of $\nu_{e}$ will be performed, while the potentials for other physics applications will be studied. In additional, an inclusive analysis of the anomalous neutrino interactions with matter will be performed.

\subsection{Scintillating CsI(Tl) Crystals}

The potential merits of crystal scintillators for low-background low-energy experiments were recently discussed $[5]$. The $\mathrm{CsI}(\mathrm{Tl})$ detector configuration for the KS experiment is displayed in Figure 3 bach crystal module is $2 \mathrm{~kg}$ in mass and consists of a hexagonalshaped cross-section with $2 \mathrm{~cm}$ side and a length $40 \mathrm{~cm}$. The light output are read out at both ends by custom-designed $29 \mathrm{~mm}$ diameter photo-multipliers (PMTs) with low-activity glass. The sum and difference of the PMT signals gives information on the energy and the longitudinal position of the events, respectively. A total of $186 \mathrm{~kg}$ (or 93 modules) have been commissioned for the Period II data taking. A major physics goal is the measurement of the Standard Model neutrino-electron scattering cross sections. The strategy [i] $\left.{ }_{1}^{0}\right]$ is to focus on data at high $(>2 \mathrm{MeV})$ recoil energy. The large target mass compensates the drop in the cross-sections.

Extensive measurements on the crystal prototype modules have been performed [i] 1 ind. The energy and spatial resolutions as functions of energy are depicted in Figure respectively. The energy is defined by the total light collection $\sqrt{\mathrm{Q}_{\mathrm{L}} * \mathrm{Q}_{\mathrm{R}}}$. It can be seen that a $\sim 10 \%$ FWHM energy resolution is achieved at $660 \mathrm{keV}$. The detection threshold (where signals are measured at both PMTs) is $<20 \mathrm{keV}$. The longitudinal position can 


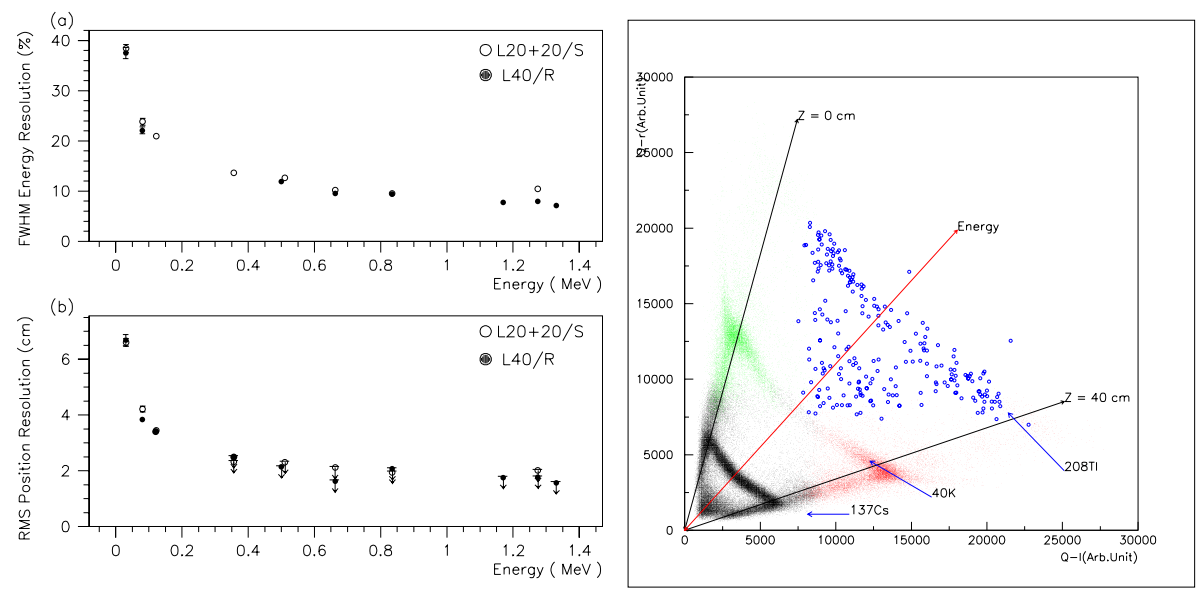

Figure 6: The variation of (a) FWHM energy resolution and (b) RMS position resolution with energy for the $\mathrm{CsI}(\mathrm{Tl})$ crystal modules. Only upper limits are shown for the higher energy points in (b) since the events are not localized. (c) $Q_{L}$ versus $Q_{R}$ distributions for single site events.

be obtained by considering the variation of the ratio $R=\left(Q_{L}-Q_{R}\right) /\left(Q_{L}+Q_{R}\right)$ along the crystal. Resolutions of $\sim 2 \mathrm{~cm}$ and $\sim 3.5 \mathrm{~cm}$ at $660 \mathrm{keV}$ and $200 \mathrm{keV}$, respectively, have been demonstrated.

In addition, $\mathrm{CsI}(\mathrm{Tl})$ provides powerful pulse shape discrimination (PSD) capabilities to differentiate $\gamma / \mathrm{e}$ from $\alpha$ events, with an excellent separation of $>99 \%$ above $500 \mathrm{keV}$. The light output for $\alpha$ 's in $\operatorname{CsI}(\mathrm{Tl})$ is quenched less than that in liquid scintillators. The absence of multiple $\alpha$-peaks above $3 \mathrm{MeV}\left[{ }_{1}^{1} \overline{1} \overline{4}\right]$ in the prototype measurements suggests that a ${ }^{238} \mathrm{U}$ and ${ }^{232} \mathrm{Th}$ concentration (assuming equilibrium) of $<10^{-12} \mathrm{~g} / \mathrm{g}$ can be achieved. It has been shown that PSD can also be achieved for pulse shapes which are partially saturated [i8].

The typical $\mathrm{Q}_{\mathrm{L}}$ versus $\mathrm{Q}_{\mathrm{R}}$ distributions for single-site events after cosmic vetos are depicted in Figure ${ }_{0}$ ic. There are evidence of contamination of internal radioactivity due to residual ${ }^{137} \mathrm{Cs}$, such that the distributions of the $662-\mathrm{keV}$ events are uniform across the $40 \mathrm{~cm}$ crystal length. Events due to $\gamma$-background from ${ }^{40} \mathrm{~K}(1460 \mathrm{keV})$ and ${ }^{208} \mathrm{Tl}$ (2612 keV), on the other hand, occur more frequently near both edges, indicating that they are from sources external to the crystals. The background is very low above $2.6 \mathrm{MeV}$, making this a favorable range to provide a measurement of $\sigma(\mathrm{SM})$.

A preliminary background energy spectrum of recoil electrons for central crystal and only one week OFF - period data sets are depicted in Figure $\bar{\tau}_{\mathbf{1}}$. For this spectrum, initially we applied moderate cuts like single event hit, single crystal hit and cosmic ray cuts. After applying these cuts we were able to see ${ }^{137} \mathrm{Cs}$ and ${ }^{40} \mathrm{~K}$ peaks. Further we applied a few specific cuts such as Alpha event cut and accidental event cut. Alpha events have very fast fall time compared to normal event and they are all located around $2.0 \mathrm{MeV}$. After applying these cuts the third peak of ${ }^{208} \mathrm{Tl}$ could be seen. In this spectrum we have not used $z$-position cuts. From this spectrum we can see that above $2.9 \mathrm{MeV}$ there are only TWO data points and there is no data point above $3.5 \mathrm{MeV}$. Thus, the signal to background ratio is $1 / 17$ above $3.0 \mathrm{MeV}$ and this ratio must be better when we calculate in term of 


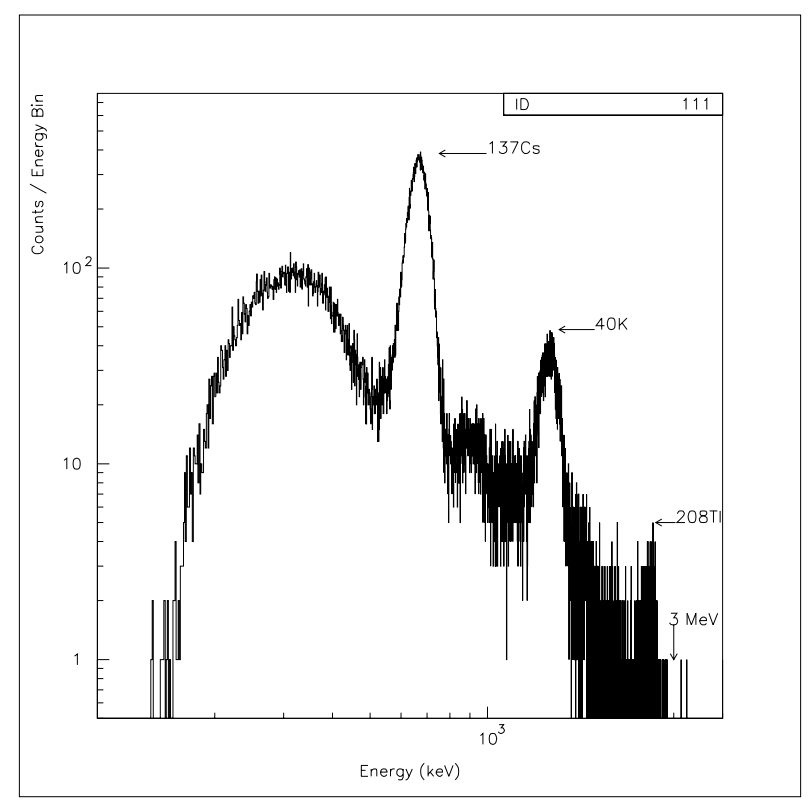

Figure 7: Background energy spectrum of recoil electrons.

ON period data over OFF period data and applying $z$-position cut.

\section{R\&D Program}

Various R\&D projects with are proceeding in parallel to the KS reactor neutrino experiment. The highlights are:

\subsection{Low Energy Neutrino Detection}

It is recognized recently that ${ }^{176} \mathrm{Yb}$ and ${ }^{160} \mathrm{Gd}$ are good candidate targets in the detection of solar neutrino $\left(\nu_{e}\right)$ by providing a flavor-specific time-delayed tag[1] Gd-loaded scintillating crystal GSO [i] $\left.\bar{i}_{\overline{6}}\right]$ indicated major background issues to be addressed. We are exploring the possibilities of developing Yb-based scintillating crystals, like doping the known crystals $\mathrm{YbAl}_{15} \mathrm{O}_{12}(\mathrm{YbAG})$ and $\mathrm{YbAlO}_{3}(\mathrm{YbAP})$ with scintillators.

The case of "Ultra Low-Energy" ULB-HPGe detectors, with the potential applications of Dark Matter searches and neutrino-nuclei coherent scatterings, is being investigated. As depicted in the measurement with a ${ }^{55} \mathrm{Fe} \mathrm{X}$-ray source on a $5 \mathrm{~g}$ prototype detector in Figure $\overline{\$}_{1}^{\prime}$, a hardware energy threshold of better than $100 \mathrm{eV}$ has been achieved. The lower energy peaks are those due to back-scattering of the X-rays from Ti. It is technically feasible to build an array of such detectors to increase the target size to the $1 \mathrm{~kg}$ mass range. 


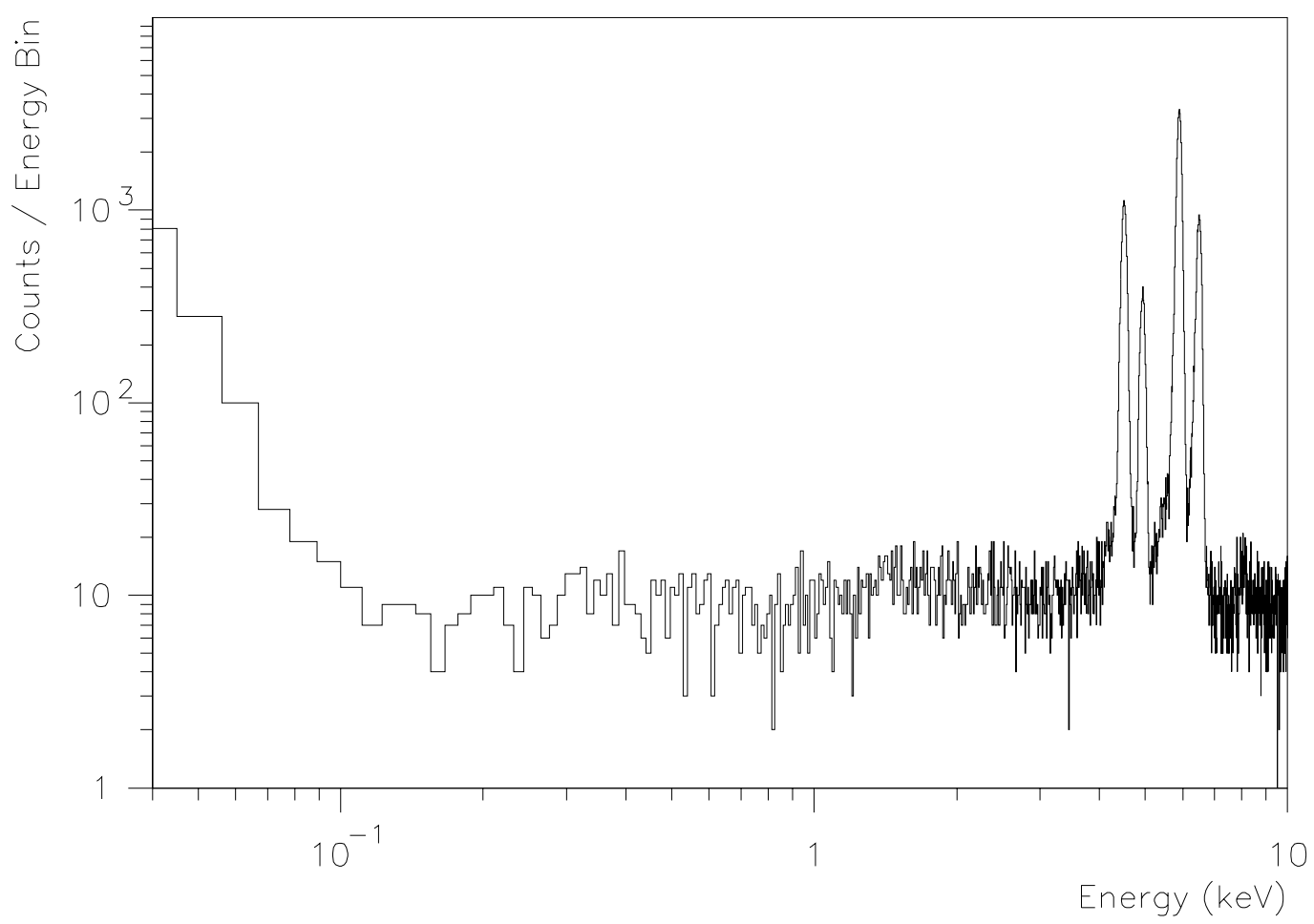

Figure 8: Measured spectrum with the ultra-low-energy HPGe detector exposed to a ${ }^{55}$ Fe source. A threshold of better than $100 \mathrm{eV}$ is achieved.

\subsection{Dark Matter Searches with CsI(Tl)}

Experiments based on the mass range of $100 \mathrm{~kg}$ of $\mathrm{NaI}(\mathrm{Tl})$ are producing some of the most sensitive results in Dark Matter "WIMP" searches[[1] $\left.\overline{7}_{1}\right]$. The feasibilities and technical details of adapting $\mathrm{CsI}(\mathrm{Tl})$ or other good candidate crystal like $\mathrm{CaF}_{2}(\mathrm{Eu})$ for WIMP Searches have been studied. A neutron test beam measurement for CsI(Tl) was successfully performed at IAE $13 \mathrm{MV}$ Tandem accelerator [1] $\left.\mathbf{1}_{1}^{\overline{8}}\right]$. We have collected the lowest threshold data for nuclear recoils in $\mathrm{CsI}(\mathrm{Tl})$, enabling us to derive the quenching factors, displayed in Figure $\overline{9}$ a a as well as to study the pulse shape discrimination techniques at the realistically low light output regime [i] $\overline{1} \bar{y}]$. The measurements also provide the first confirmation of the Optical Model predictions on neutron elastic scatterings with a direct measurement on the nuclear recoils of heavy nuclei, as illustrated by the differential cross-section measurements of Figure $\bar{p}$. A full scale Dark Matter experiment with $\mathrm{CsI}(\mathrm{Tl})$ crystals is being pursued by the KIMS Collaboration in South Korea[2] $[\overline{0}]$.

\subsection{Radio-purity Measurements with Accelerator Mass Spectrometry}

Measuring the radio-purity of detector target materials as well as other laboratory components are crucial to the success of low-background experiments. The typical methods are direct photon counting with high-purity germanium detectors, $\alpha$-counting with silicon detectors, conventional mass spectrometry or the neutron activation techniques. We are 
a)

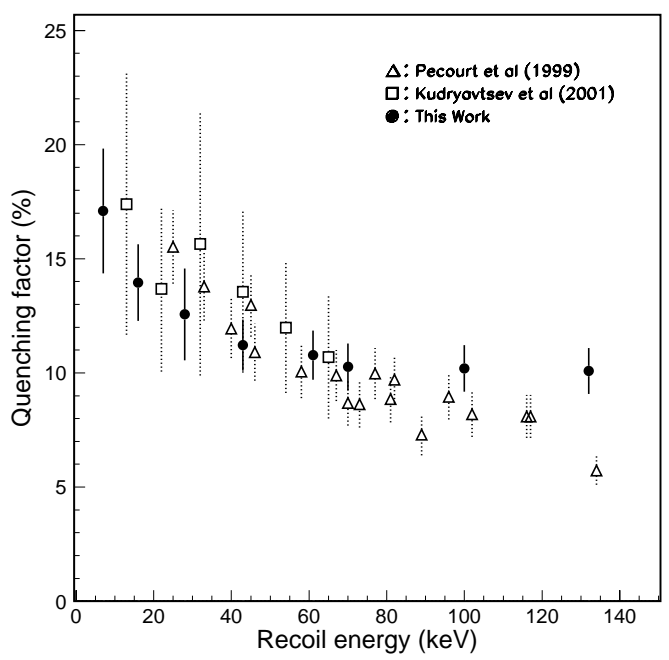

b)

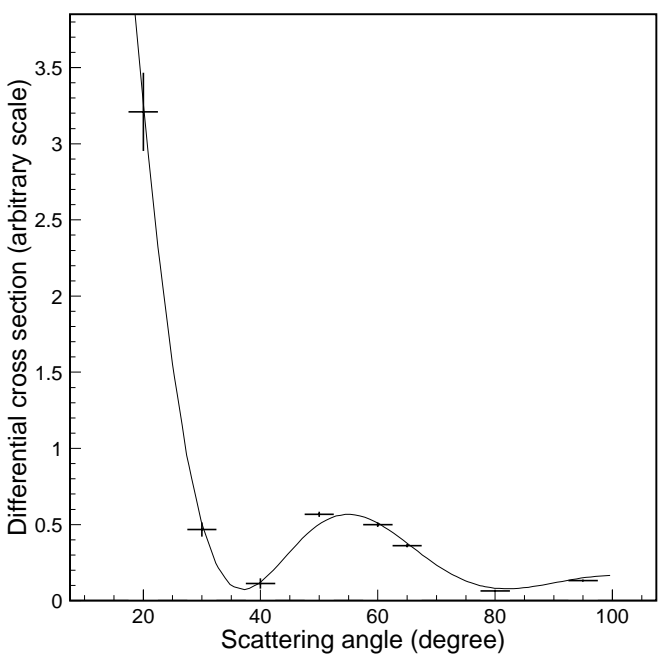

Figure 9: (a) The quenching factors, shown as black circles, measured at IAE Tandem, as compared to previous work. (b) The measured nuclear recoil differential cross sections in $\mathrm{CsI}(\mathrm{Tl})$, superimposed with the Optical Model predictions.

exploring the capabilities of radio-purity measurements further with the new Accelerator

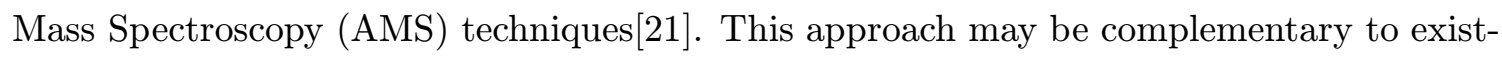
ing methods since it is in principle a superior and more versatile method as demonstrated in the ${ }^{13} \mathrm{C}$ system, and it is sensitive to radioactive isotopes that do not emit $\gamma$-rays (like single beta-decays from ${ }^{87} \mathrm{Rb}$ and ${ }^{129} \mathrm{I}$ ) or where $\gamma$ emissions are suppressed (for instance, measuring ${ }^{39} \mathrm{~K}$ provides a gain of $10^{5}$ in sensitivity relative to detecting $\gamma$ 's from ${ }^{40} \mathrm{~K}$ ). A pilot measurement of the ${ }^{129} \mathrm{I} /{ }^{127} \mathrm{I}$ ratio $\left(<10^{-12}\right)$ in CsI was successfully performed demonstrating the capabilities of the Collaboration. Further beam time is scheduled at the IAE AMS facilities[2] $\overline{2} \overline{2}]$ to devise measuring schemes for the other other candidate isotopes like ${ }^{238} \mathrm{U},{ }^{232} \mathrm{Th},{ }^{87} \mathrm{Rb},{ }^{40} \mathrm{~K}$ in liquid and crystal scintillators beyond the present capabilities by the other techniques. The first isotope to study is on ${ }^{40} \mathrm{~K}$, where the goal sensitivity of a $10^{-14} \mathrm{~g} / \mathrm{g}$ should be achieve-able by the AMS techniques.

\subsection{Upgrade of FADC for LEPS Experiment}

Based on the design and operation of the FADCs at the KS experiment, we developed new FADCs for the Time Projection Chamber (TPC) constructed as a sub-detector for the

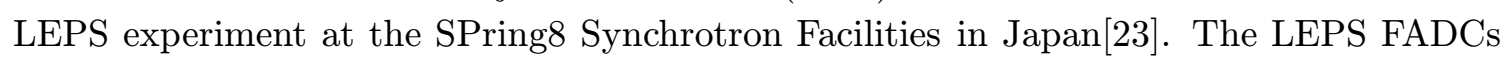
have $40 \mathrm{MHz}$ sampling rate, 10-bit dynamic range, 32 channels per module and are equipped with Field Programmable Gate Array (FPGA) capabilities for real time data processing. The TPC has 1000 readout channels and a typical cosmic-ray event is depicted in Figure $10_{1}^{1}$ The new system will be commissioned at LEPS in summer 2003. The upgraded FADCs will be further optimized and implemented to the KS reactor neutrino experiment for data taking in 2004. 


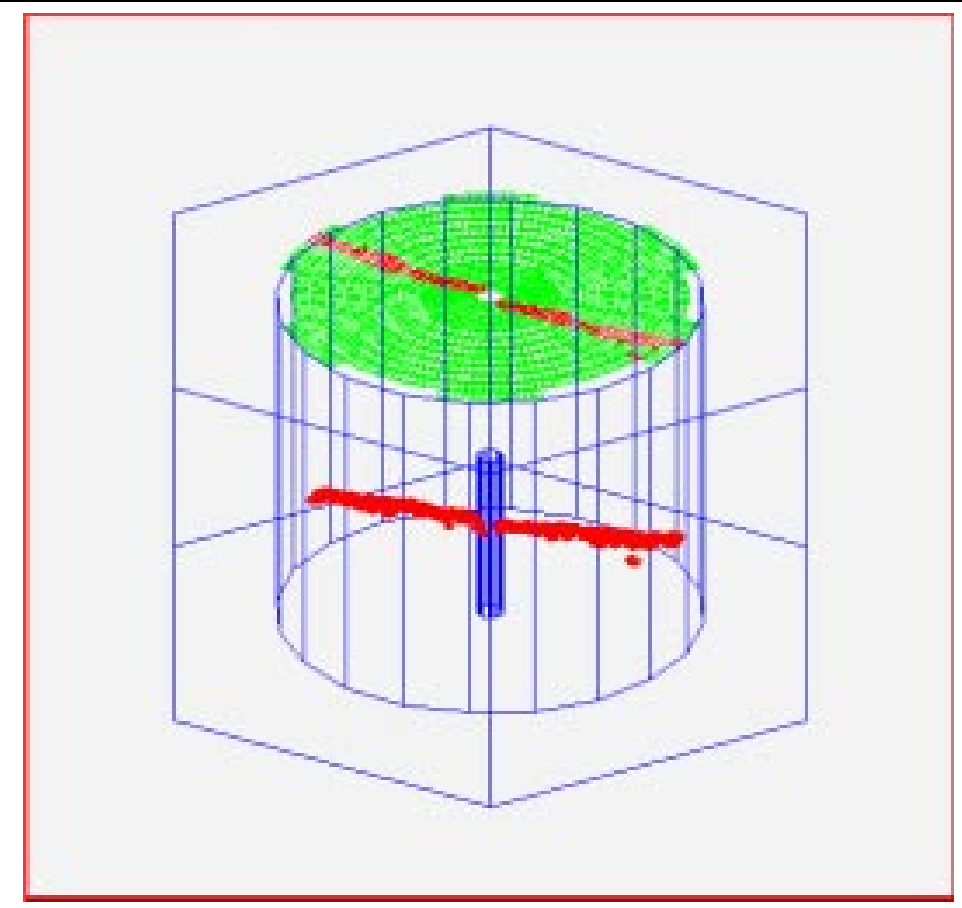

Figure 10: A typical cosmic-ray event recorded by the TPC for the LEPS Experiment, using an FADC-based data acquisition system.

\section{Outlook}

The strong evidence of neutrino masses and mixings [it] lead to intense world-wide efforts to pursue the next-generation of neutrino projects. Neutrino physics and astrophysics will remain a central subject in experimental particle physics in the coming decade and beyond. There are room for ground-breaking technical innovations - as well as potentials for surprises in the scientific results.

A collaboration among scientists from Taiwan and China has been built up with the goal of establishing a qualified experimental program in neutrino and astro-particle physics. It is the first generation collaborative efforts in large-scale basic research between scientists from Taiwan and China. The flagship effort is to perform the first-ever particle physics experiment in Taiwan at the Kuo-Sheng Reactor Plant. World-level sensitivities on the neutrino magnetic moment and radiative lifetime have already been achieved with the Period I data using a high-purity germanium detector. Further measurements are pursued at the Kuo-Sheng Laboratory, including the Standard Model neutrino-electron scattering cross-section as well as neutrino coherent scattering with the nuclei. A wide spectrum of $\mathrm{R} \& \mathrm{D}$ projects are being pursued in parallel.

The importance of the implications and outcomes of the experiment and experience will lie besides, if not beyond, neutrino physics.

\section{Acknowledgments}

The authors are grateful to the scientific members, technical staff and industrial partners of TEXONO Collaboration, as well as the concerned colleagues in our communities for the 
many contributions which "make it happen" in such a short period of time. Funding are provided by the National Science Council, Taiwan and the National Science Foundation, China, as well as from the operational funds of the collaborating institutes.

\section{References}

[1] Home Page at http://hepmail.phys.sinica.edu.tw/ texono/

[2] C.Y. Chang, S.C. Lee and H.T. Wong, Nucl. Phys. B (Procs. Suppl.) 66, 419 (1998).

[3] D. Normile, Science 300, 1074 (2003).

[4] See the respective sections in Review of Particle Physics, Particle Data Group, Phys. Rev. D 66 (2002), for details and references.

For recent updates, see Proc. of the XXth Int. Conf. on Neutrino Physics \& Astrophysics, eds. F. von Feilitzsch and N. Schmitz, Nucl. Phys. B (Proc. Suppl.) 118 (2003).

[5] H.T. Wong et al., Astropart. Phys. 14, 141 (2000).

[6] H.T. Wong and J. Li, Mod. Phys. Lett. A 15, 2011 (2000);

H.B. Li et al., TEXONO Coll., Nucl. Instrum. Methods A 459, 93 (2001).

[7] W.P. Lai et al., TEXONO Coll., Nucl. Instrum. Methods A 465, 550 (2001).

[8] Q. Yue et al., Nucl. Instrum. Methods A 511, 408 (2003).

[9] B. Kayser et al., Phys. Rev. D 20, 87 (1979); P.Vogel and J.Engel, Phys. Rev. D 39, 3378 (1989).

[10] H.B. Li and H.T. Wong, J. Phys. G 28, 1453 (2002).

[11] H.B. Li et al., TEXONO Coll., Phys. Rev. Lett. 90, 131802 (2003).

[12] G.G. Raffelt, Phys. Rev. D 39, 2066 (1989).

[13] Y. Liu et al., TEXONO Coll., Nucl. Instrum. Methods A 482, 125 (2002).

[14] U. Kilgus, R. Kotthaus, and E. Lange, Nucl. Instrum. Methods A 297, 425, (1990);

R. Kotthaus, Nucl. Instrum. Methods A 329, 433 (1993).

[15] R.S. Raghavan, Phys. Rev. Lett. 78, 3618 (1997).

[16] S.C. Wang, H.T. Wong, and M. Fujiwara, Nucl. Instrum. Methods A 479, 498 (2002).

[17] R. Bernabei et al., Phys. Lett.B 480, 23 (2000), and references therein.

[18] M.Z. Wang et al., Phys. Lett. B 536, 203 (2002).

[19] S.C. Wu et al., physics/0307002, submitted to Nucl. Instrum. Methods A (2003).

[20] H.J. Kim et al., Nucl. Instrum. Methods A 457, 471 (2001).

[21] D. Elmore and F.M. Phillips, Science 346, 543 (1987).

[22] S. Jiang et al., Nucl. Instrum. Methods B 52, 285 (1990);

S. Jiang et al., Nucl. Instrum. Methods B 92, 61 (1994).

[23] T. Nakano, LEPS Coll., Nucl. Phys. A 684, 71c (2001). 